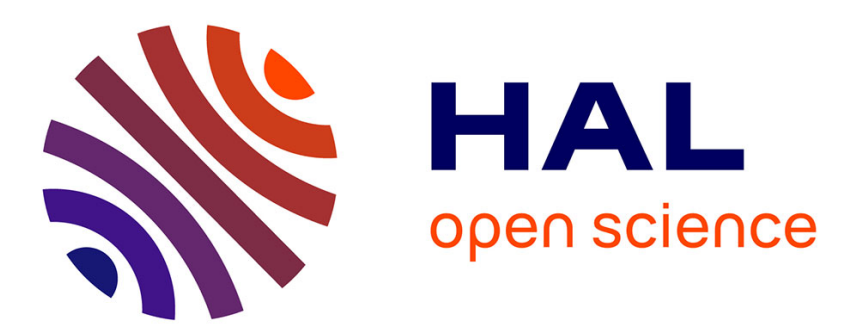

\title{
Extended-Forward Architecture for Simplified Check Node Processing in NB-LDPC Decoders
}

Cédric Marchand, Emmanuel Boutillon, Hassan Harb, Laura Conde-Canencia, Ali Al Ghouwayel

\section{- To cite this version:}

Cédric Marchand, Emmanuel Boutillon, Hassan Harb, Laura Conde-Canencia, Ali Al Ghouwayel. Extended-Forward Architecture for Simplified Check Node Processing in NB-LDPC Decoders. SIPS'2017, Oct 2017, Lorient, France. hal-01613799

\section{HAL Id: hal-01613799 \\ https://hal.science/hal-01613799}

Submitted on 10 Oct 2017

HAL is a multi-disciplinary open access archive for the deposit and dissemination of scientific research documents, whether they are published or not. The documents may come from teaching and research institutions in France or abroad, or from public or private research centers.
L'archive ouverte pluridisciplinaire HAL, est destinée au dépôt et à la diffusion de documents scientifiques de niveau recherche, publiés ou non, émanant des établissements d'enseignement et de recherche français ou étrangers, des laboratoires publics ou privés. 


\section{Extended-Forward Architecture for Simplified Check Node Processing in NB-LDPC Decoders}

\author{
Cédric Marchand, Emmanuel Boutillon, \\ Hassan Harb and Laura Conde-Canencia \\ Lab-STICC, CNRS UMR 6285 \\ Université de Bretagne Sud, Lorient, France \\ Email: emmanuel.boutillon@univ-ubs.fr
}

\author{
Ali Al Ghouwayel \\ CCE Department \\ Lebanese International University (LIU), Beirut, Lebanon. \\ Email: ali.ghouwayel@liu.edu.lb
}

\begin{abstract}
This paper focuses on low complexity architectures for check node processing in Non-Binary LDPC decoders. To be specific, we focus on Extended Min-Sum decoders and consider the state-of-the-art Forward-Backward and SyndromeBased approaches. We recall the presorting technique that allows for significant complexity reduction at the Elementary Check Node level. The Extended-Forward architecture is then presented as an original new architecture for efficient syndrome calculation. These advances lead to a new architecture for check node processing with reduced area. As an example, we provide implementation results over GF(64) and code rate 5/6 showing complexity reduction by a factor of up to 2.6 .
\end{abstract}

Index Terms-NB-LDPC, Check Node, Syndrome-Based, Foward-Backward, VLSI.

\section{INTRODUCTION}

Low-Density Parity-Check (LDPC) codes [1] have now been adopted for a wide range of standards (WiMAX, WiFi, DVB-C2, DVB-S2X, DVB-T2) because of their near-channelcapacity performance. However, this performance is mainly obtained for long codeword lengths. The last decade witnessed a great deal of research effort devoted to the extended version of LDPC codes defined over ( $\mathrm{GF}(q), q>2)$. These codes called Non-Binary (NB) LDPC codes have shown strong potential in error correction capability with moderate and short codeword lengths [2]. NB-LDPC codes retain the benefits of steep waterfall region (typical of convolutional turbo-codes) and low error floor (typical of binary LDPC). Compared to their binary counterparts, NB-LDPC codes generally present higher girths, which leads to better decoding performance. Moreover, the NB nature of such NB-LDPC codes makes them suitable for high-spectral-efficiency modulation schemes where the constellation symbols are directly mapped to $\operatorname{GF}(q)$ symbols. This mapping bypasses the marginalization process of binary LDPC codes that causes information loss. These characteristics place NB-LDPC codes as serious competitors of classical binary LDPC and Turbo-Codes in future wireless communication and digital video broadcasting standards. However, NB-LDPC codes suffer from high decoding complexity. In the NB decoder each message exchanged between processing nodes is an array of values, each value corresponding to a GF element. From an implementation point of view, this leads to a highly increased complexity compared to binary LDPC.
TABLE I

CN ARCHITECTURES BASED ON THE EMS ALGORITHM.

\begin{tabular}{c|c||c|c}
\hline \multicolumn{2}{c||}{ CN architectures } & \multicolumn{2}{c}{ Input processing } \\
\hline Abbreviation & Name & Normal & PreSorted \\
\hline \hline T-EMS & Trellis EMS & {$[6]$} & - \\
\hline FB & Forward-Backward & {$[4],[5],[9]$} & {$[10]^{*}$} \\
\hline SB & Syndrome-Based & {$[7]^{*},[8]^{*},[11]$} & {$[12]^{*}$} \\
\hline EF & Extended Forward-SB & This paper & This paper \\
\hline
\end{tabular}

The extension of the well-known binary Min-Sum algorithm to the NB domain, called Extended Min-Sum (EMS), represents an interesting compromise between hardware complexity and error correction performance [3], [4]. The computational bottleneck in the EMS algorithm is the Check Node (CN) processing. State-of-the-art architectures apply the ForwardBackward (FB) algorithm [4], [5] to efficiently implement the CN. The FB approach uses Elementary Check Nodes (ECN) in a 3-layer structure to reduce the hardware cost of the $\mathrm{CN}$ as well as the number of computations, as it is possible to reuse intermediate results. However, the FB CN structure suffers from high latency and low throughput rate. The TrellisEMS (T-EMS) introduced in [6] reduces the latency of the FB computation but presents a hardware complexity that significantly increases with $q$ when a parallel implementation is considered. The Syndrome-Based Check Node (SB CN) algorithm, recently presented in [7] [8], is an efficient method to perform in parallel the $\mathrm{CN}$ computations for high order Galois fields $(q \geq 16)$. However, the complexity of the SB $\mathrm{CN}$ algorithm is dominated by the number of syndromes to be computed, which increases quadratically with $d_{c}$. This limits its interest for high coding rates, i.e. high $d_{c}$ values.

Recently, we showed that sorting the input vector of the $\mathrm{CN}$ according to a reliability criterion [12] [10] allows significant reduction of the hardware complexity of the $\mathrm{CN}$ architecture without affecting the performance. This technique, named presorting, has been successfully combined with the SB CN leading to the PreSorted SB CN (or PS-SB CN) architecture [12] and with the FB CN to give the PS-FB CN [10]. Table I summarizes the different $\mathrm{CN}$ architectures based on the EMS algorithm and introduces the architecture proposed in this paper, i.e. the Extended-Forward CN, with and without 
application of the presorting technique. The references with an asterisk correspond to previous contributions of the authors.

The rest of the paper is organized as follows: Section II briefly introduces NB-LDPC codes as well as the Min-Sum and the Extended Min-Sum algorithms. It also reminds the principles of the Forward-Backward and the Syndrome-Based $\mathrm{CN}$ architectures. Section III presents the new ExtendedForward approach and how the presorting technique can lead to a more efficient architecture. Performance and synthesis results are presented in Section IV, for comparison with the state-of-the-art architectures. Finally, section V concludes the work.

\section{NB-LDPC CODES AND EXTENDED MIN-SUM DECODING}

This Section first introduces NB-LDPC codes and their notations. NB-LDPC decoding is then considered with the description of the Min-Sum algorithm and its extended version.

\section{A. NB-LDPC codes}

An NB-LDPC code is a linear block code defined over a Galois Field of size $q, \mathrm{GF}(q)$, and with a sparse parity-check matrix $\mathbf{H}$ of size $M \times N$. The $M$ rows of the matrix $\mathbf{H}$ refer to $M$ parity-check equations. The $i^{t h}$ parity-check equation is expressed as

$$
\sum_{k=1}^{d_{c}(i)} h_{i, j_{i}(k)} x\left(j_{i}(k)\right)=0
$$

where $d_{c}(i)$ in the number of non-zero $\operatorname{GF}(q)$ values which are denoted as $\left\{h_{i, j_{i}(k)}\right\}_{k=1 \ldots d_{c}(i)}$ and $\left\{x\left(j_{i}(k)\right)\right\}_{k=1 \ldots d_{c}(i)}$ is a subset of size $d_{c}(i)$ of the $N \mathrm{GF}(q)$ symbols of the code. A NB-LDPC code is regular if the number $d_{c}$ of non-zero values per row and the number $d_{v}$ of non-zero values per column are constant. The authors in [13] showed that robust NB-LDPC codes can be constructed with $d_{v}=2$. In that case, assuming a full rank parity check matrix $\mathbf{H}$, the rate of the code is $r=1-2 / d_{c}$. An $(M, N) \mathrm{GF}(q)$ NB-LDPC code has a binary counterpart of size $(M \times m, N \times m)$ with $m=\log _{2}(q)$.

\section{B. Min-Sum algorithm}

For simplicity, the algorithms are described only at the $\mathrm{CN}$ level. The reader can refer to [14] for a complete description of the variable node and edge node processors.

Let us define a $\mathrm{CN}$ equation of degree $d_{c}$ in $\mathrm{GF}(q)$ as $e_{1} \oplus$ $e_{2} \oplus e_{3} \oplus \ldots \oplus e_{d_{c}}=0$ where the operator $\oplus$ represents addition over $\operatorname{GF}(q)$. Fig. 1 shows a $\mathrm{CN}$ for $d_{c}=4$ and the associated messages. Inputs $e_{i}, i \in 1, \ldots, d_{c}$ take their values on the alphabet of size $q$. The a priori information about variable $e$ is the discrete probability distribution $P(e=x), x \in \mathrm{GF}(q)$. Each element of the probability distribution $E$ associated to $e$ can be expressed in the $\log$ domain as the Log Likelihood Ratio (LLR) $e^{+}(x)$ defined as

$$
e^{+}(x)=-\log \left(\frac{P(e=x)}{P(e=\bar{x})}\right)
$$

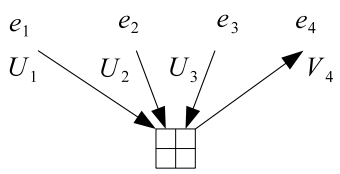

Fig. 1. Message notation on a $\mathrm{CN}$

where $\bar{x}$ is the hard decision on $e$ obtained by taking the most probable GF symbol, i.e. $\bar{x}=\arg \max _{x \in \mathrm{GF}(q)} P(e=x)$. By definition of the LLR, we have: $e^{+}(\bar{x})=0$ and $\forall x \in \operatorname{GF}(q)$, $e^{+}(x) \geq 0$. The distribution (or message) $E$ associated to $e$ is thus $E=\left\{e^{+}(x)\right\}_{x \in \mathrm{GF}(q)}$.

The Min-Sum algorithm computes the LLR value of the $i^{\text {th }}$ output for the GF symbol $x$ as $v_{i}^{+}(x)$

$$
v_{i}^{+}(x)=\min \left\{\sum_{i^{\prime}=1, i^{\prime} \neq i}^{d_{c}} e_{i^{\prime}}^{+}\left(x_{i^{\prime}}\right) \mid \bigoplus_{i^{\prime}=1, i^{\prime} \neq i}^{d_{c}} x_{i^{\prime}}=x\right\},
$$

where $x_{i^{\prime}} \in \mathrm{GF}(q)$ for $i^{\prime}=1, \ldots, d_{c}, i^{\prime} \neq i$.

\section{EMS algorithm}

The main characteristic of the EMS algorithm is to truncate message $E$ from $q$ values to the $n_{m}$ most reliable ones, with $n_{m} \ll q$. The resulting message $U$ is composed of $n_{m}$ couples sorted in increasing order of LLRs (the higher the LLR value, the lower the reliability). Fig. 1 illustrates the principle of $\mathrm{CN}$ processing. Input $U$ of a $\mathrm{CN}$ is a list $\{U[j]\}_{j=0 \ldots n_{m}-1}$ of couples $U[j]=\left(U^{+}[j], U^{\oplus}[j]\right)$, where $U^{+}[j]$ denotes the $j^{t h}$ smallest LLR value of $E$ and $U^{\oplus}[j]$ denotes its associated GF element, i.e., $e^{+}\left(U^{\oplus}[j]\right)=U^{+}[j]$. Note also that $U^{+}[0]=0$, $U^{\oplus}[0]=\bar{x}$, and that $j \leq j^{\prime} \Rightarrow U^{+}[j] \leq U^{+}\left[j^{\prime}\right]$. The same representation is used for each output $V$ of a $\mathrm{CN}$.

The EMS algorithm is a simplification of the Min-Sum algorithm and can be described in two steps:

1) Evaluate: eq. (2) is modified by replacing $x_{i^{\prime}} \in \mathrm{GF}(q)$ by $x_{i^{\prime}}$ in the set of available GF data, i.e., $x_{i^{\prime}} \in U_{i}^{\oplus}$, with $U_{i}^{\oplus}=\left\{U_{i}^{\oplus}[j]\right\}_{j=0,1, \ldots, n_{m}-1}$ :

$$
v_{i}^{+}(x)=\min \left\{\sum_{i^{\prime}=1, i^{\prime} \neq i}^{d_{c}} U_{i^{\prime}}^{+}\left[j_{i^{\prime}}\right] \mid \bigoplus_{i^{\prime}=1, i^{\prime} \neq i}^{d_{c}} U_{i^{\prime}}^{\oplus}\left[j_{i^{\prime}}\right]=x\right\},
$$

where $j_{i^{\prime}} \in\left\{0,1, \ldots n_{m}-1\right\}$ for $i^{\prime}=1,2, \ldots d_{c}, i^{\prime} \neq i$.

2) Sort: the $v_{i}^{+}(x)$ are sorted in increasing order and the first $n_{m}$ smallest values are kept to generate the output vector $V_{i}$.

\section{Forward-Backward CN}

As described in [4], the FB consists of three layers of $d_{c}-2$ ECNs each. An ECN processes a single output $C$ as a function of two inputs $A$ and $B$. Intermediate results of the ECNs are reused in the later stages to avoid re-computations, thus reducing the amount of processing to generate each output message.

The ECN processing [15] can be described in three steps. 


\begin{tabular}{|c|c|c|c|}
\hline$U_{1}$ & $U_{2}$ & $U_{3}$ & $U_{4}$ \\
\hline$\left(5, \alpha^{32}\right)$ & $\left(7, \alpha^{12}\right)$ & $\left(4, \alpha^{41}\right)$ & $\left(6, \alpha^{59}\right)$ \\
\hline$\left(4, \alpha^{3}\right)$ & $\left(6, \alpha^{45}\right)$ & $\left(3, \alpha^{33}\right)$ & $\left(5, \alpha^{19}\right)$ \\
\hline$\left(3, \alpha^{21}\right)$ & $\left(5, \alpha^{61}\right)$ & $\left(2, \alpha^{18}\right)$ & $\left(4, \alpha^{46}\right)$ \\
\hline$\left(2, \alpha^{16}\right)$ & $\left(5, \alpha^{41}\right)$ & $\left(1, \alpha^{17}\right)$ & $\left(3, \alpha^{51}\right)$ \\
\hline$\left(0, \alpha^{5}\right.$ & $\left(0, \alpha^{21}\right)$ & $\left(0, \alpha^{21}\right)$ & $\left(0, \alpha^{15}\right)$ \\
\hline
\end{tabular}

$\delta=(0,1,0,2) \quad S(\delta)=\left(9, \alpha^{42}, 0101\right)$

Fig. 2. Example of a deviation path

1) Addition: For each couple of index $(a, b) \in$ $\left\{0,1, \ldots, n_{m}-1\right\}^{2}$, the output tuple $C_{a, b}=\left(C^{+}(x), x\right)$ is computed as

$$
C_{a, b}=\left(A^{+}[a]+B^{+}[b], A^{\oplus}[a] \oplus B^{\oplus}[b]\right)
$$

2) Sorting: The couples $\left(c^{+}(x), x\right)$ are sorted in increasing order of $c^{+}(x)$.

3) Redundancy Elimination (RE): In case of two couples with the same GF value, the one with the higher LLR is suppressed during this RE step.

For the sake of clarity, the three ECN steps are represented using the symbol $\boxplus$ and the ECN equation is

$$
C=A \boxplus B
$$

\section{E. Syndrome-Based CN}

The SB CN algorithm [7] relies on the definition of a deviation path and its associated syndrome. In the sequel, $n_{m, \text { in }}$ (resp. $n_{m, o u t}$ ) refers to the size of the input (resp. output) vector of a $\mathrm{CN}$.

A deviation path, denoted by $\delta$, is defined as a $d_{c^{-}}$ tuple of integer values between 0 and $n_{m, i n}-1$, i.e. $\delta=$ $\left(\delta(1), \delta(2), \ldots, \delta\left(d_{c}\right)\right)$, with $\delta(i) \in\left\{0,1, \ldots, n_{m, i n}-1\right\}$, $i=1,2 \ldots, d_{c}$.

The SB considers a set $\Delta$ of deviation paths on the $d_{c}$ input messages to compute associated syndromes.

The syndrome $S(\delta)$ associated to deviation path $\delta$ is defined by the 3-tuple $\left(S^{+}(\delta), S^{\oplus}(\delta), S^{D}(\delta)\right)$, with

$$
\begin{gathered}
S^{+}(\delta)=\sum_{i=1}^{d_{c}} U_{i}^{+}[\delta(i)], S^{\oplus}(\delta)=\bigoplus_{i=1}^{d_{c}} U_{i}^{\oplus}[\delta(i)] \text { and } \\
S^{D}(\delta)[i]= \begin{cases}0, & \text { if } \delta(i)=0 \\
1, & \text { otherwise }\end{cases}
\end{gathered}
$$

where $S^{+}(\delta)$ is an LLR value, $S^{\oplus} \in \mathrm{GF}(q)$, and $S^{D}(\delta)$ is a binary vector of size $d_{c}$ called Discard Binary Vector (DBV).

An example of deviation path $\delta$ and its associated syndrome $S(\delta)$ is shown in Fig. 2.

After syndromes computation, they are sorted and then decorrelated for each output message $V_{i}, i=1, \ldots, d_{c}$. The $\mathrm{SB} \mathrm{CN}$ architecture is given in Fig. 3. In particular, decorrelation is performed by $d_{c}$ Decorrelation Units (DU). DUs are represented in parallel to show the inherent parallelism of

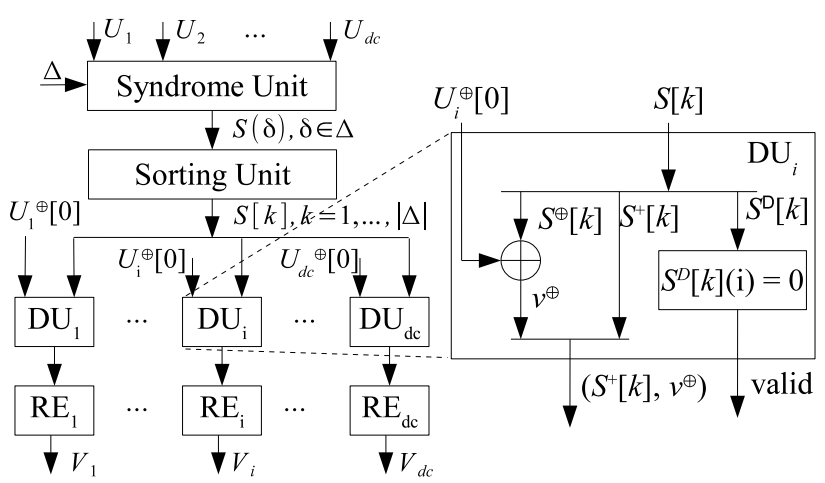

Fig. 3. Syndrome-based CN processing (left part), details of the DU unit (right part)

the SB CN. Fig. 3 also shows the schematic operations of the DUi. $S^{D}$ is the $d_{c}$-wide bit vector that indicates for which output edges the syndrome should be discarded during the decorrelation process. A simple reading of bit $i$ in the binary vector $S^{D}$ validates or not the syndrome for the output edge $i$.

\section{F. Presorting technique}

The presorting technique consists in permuting the input vectors as a function of $U_{i}^{+}[1], i=1,2, \ldots, d_{c}$. Fig. 4 illustrated an application of the presorting algorithm on the EMSbased $\mathrm{CN}$, where $n_{m}=4$ and $d_{c}=4$. In this particular example, the hatched tuples represent tuples that are not involved in the computation of the first 8 syndromes. Compared to the standard $\mathrm{CN}$, the presorting process requires extra hardware: a $d_{c}$-input vector sorter and two permutation networks (or switches). However, it allows some simplifications in the $\mathrm{CN}$ itself, globally leading to a complexity reduction of the whole $\mathrm{CN}$ processing. The presorting technique was applied to both FB [10] and SB [12], leading to significant complexity reductions in the decoder $\mathrm{CN}$ architectures.

\section{NEW CN ARCHITECTURE: SYNDROME COMPUTATION WITH EXTENDED-FORWARD PROCESSING}

In this section, we present an architecture that dynamically generates a set of syndromes, keeping the complexity linear with $d_{c}$. The output of a forward layer in a FB decoder processes all input except last one $U_{d_{c}}$. The idea is to add an ECN to merge the forward layer with $U_{d_{c}}$. The output of this Extended Forward (EF) generates in fact a set of sorted syndromes $S_{b}$. The syndrome computation is thus equivalent to the equation

$$
S_{b}=\boxplus_{i=1}^{d_{c}} U_{i} .
$$

The associated EF CN architecture with $d_{c}=6$ is presented in Fig. 5. Thus $d_{c}-1$ ECNs with parameters $\left(n_{m}, n_{m}, n_{m}\right)$ will provide $n_{m}$ syndromes sorted in increasing order of their associated LLR values.

$S_{b}$ can also be computed in $\log _{2}\left(d_{c}\right)$ layers of ECNs using a tree structure as shown in Fig. 6 or in any semi-parallel 


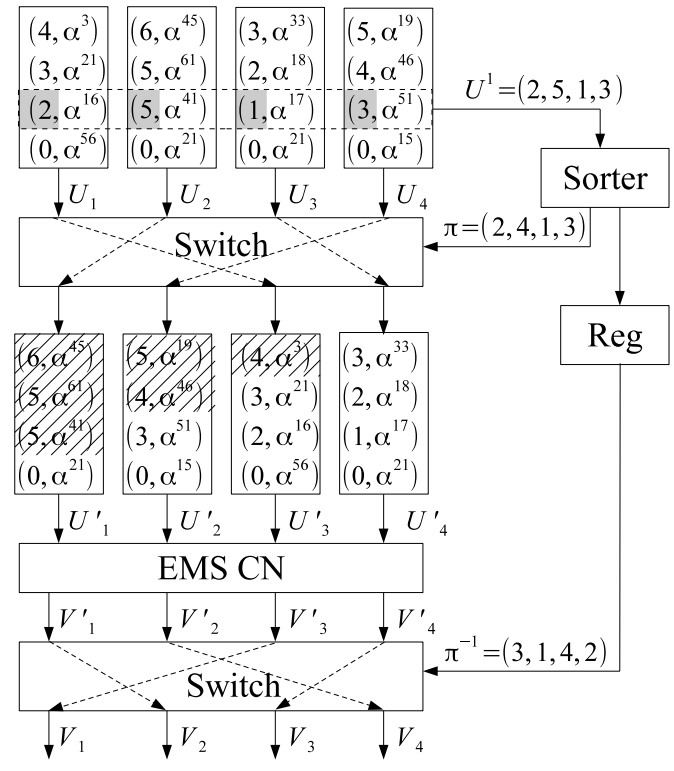

Fig. 4. Presorting principle applyed on an EMS-based CN architecture

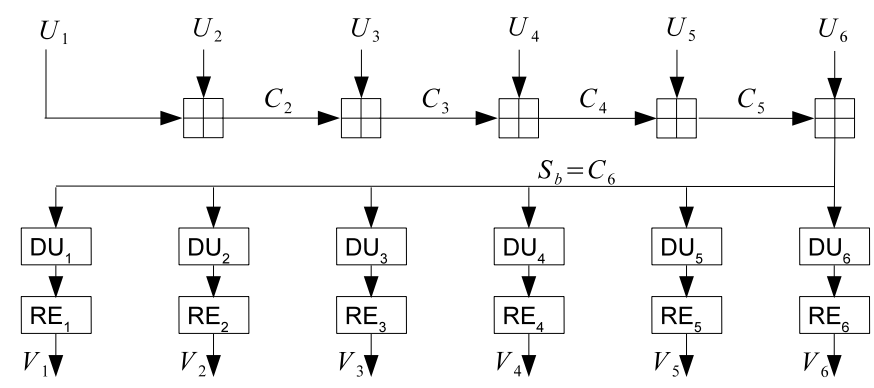

Fig. 5. EF CN Architecture

compromise. As shown in Fig. 5 and Fig. 6 the complexity is of $d_{c}-1$ ECNs and is thus linear with $d_{c}$.

\section{A. Construction of the Discard Binary Vector}

Thanks to the use of ECNs, the computed syndrome set is sorted and can be directly applied to the decorrelation process.

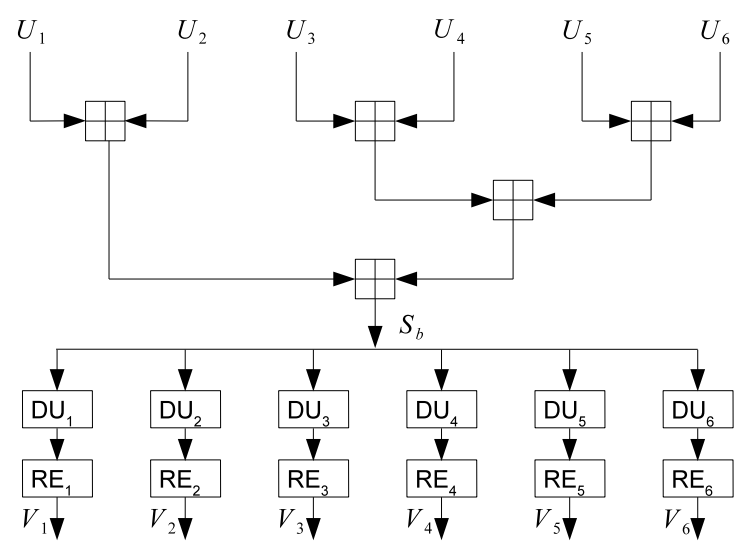

Fig. 6. EF CN with tree architecture
However, each ECN must perform an additional operation to generate a new term $c_{a, b}^{D}$, that is needed to construct the DBV $S^{D}$ required in the decorrelation process ( (7) and right part of Fig. 3). The ECN addition is modified as

$$
\begin{aligned}
C_{a, b}=\left(c_{a, b}^{+}, c_{a, b}^{\oplus}, c_{a, b}^{D}\right)= & \left(A^{+}[a]+B^{+}[b],\right. \\
& A^{\oplus}[a] \oplus B^{\oplus}[b], \\
& \left.A^{D}[a] \| B^{D}[b]\right)
\end{aligned}
$$

where $\|$ represents the concatenation operation of two binary vectors.

In the $\mathrm{EF}$ processing, the $\mathrm{CN}$ inputs are initialized with a DBV value of one bit, as follows: $U_{i}^{D}[j]=1, \forall j \neq 0$ and $U_{i}^{D}[0]=0$. Thanks to the DBV computation, the output of the EF processing is similar to (6) and (7) with the only difference that the set of deviation paths $\Delta_{E F}$ is input dependent, while it is predefined offline for the SB [7], [8].

\section{B. The effect of RE in ECNs}

In the EF processing, the number of computed syndromes is typically $3 \times n_{m, \text { out }}$ to compensate for the discarded redundant syndromes [7], [8]. However, even with this approach, the first simulation results of the EF algorithm showed significant performance degradation compared to the FB algorithm (red curve in Fig. 8). The reason of this performance degradation resides in the RE step performed by each ECN: since each ECN performs RE, no more than one syndrome can be associated to a given GF value. Let us consider two syndromes with same GF value $x$ and different deviation paths at the input of a DU where the second syndrome is validated by the DU and not the first one. If the second (valid) syndrome has been discarded previously by a RE then no syndrome with GF value $x$ can be validated and the default maximum LLR is associated with $x$ leading to performance degradation. The solution for this problem is to make sure that there is no RE before DU and to perform RE after DU as shown in Fig. 5 and Fig. 6.

\section{Extended Forward $\mathrm{CN}$ with presorting}

The presorting technique leads to significant hardware savings by reducing the number of candidate GF symbols in the ECNs [10]. This technique can also be applied to the $\mathrm{EF} \mathrm{CN}$ architecture for complexity reduction thanks to the limited number of bubbles to be considered in each ECN. For this, we perform a statistical study based on MonteCarlo simulation that traces the paths of the GF symbols that contribute to the output of the $\mathrm{CN}$, on their way across the different ECNs. With this information we know, for each ECN, how often a specific bubble contributes to an output and we can then prune the bubbles that never or rarely contribute to an output. A short simulation on less than 10000 frames is more than enough to produce reliable statistics on the bubble order of importance. However, the best method on how to prune low-score bubbles is still an open question. We obtained good results by cumulating the sorted bubble scores and discard bubbles above a threshold given by a percentage of the total cumulated scores. In a second step, bubbles are 


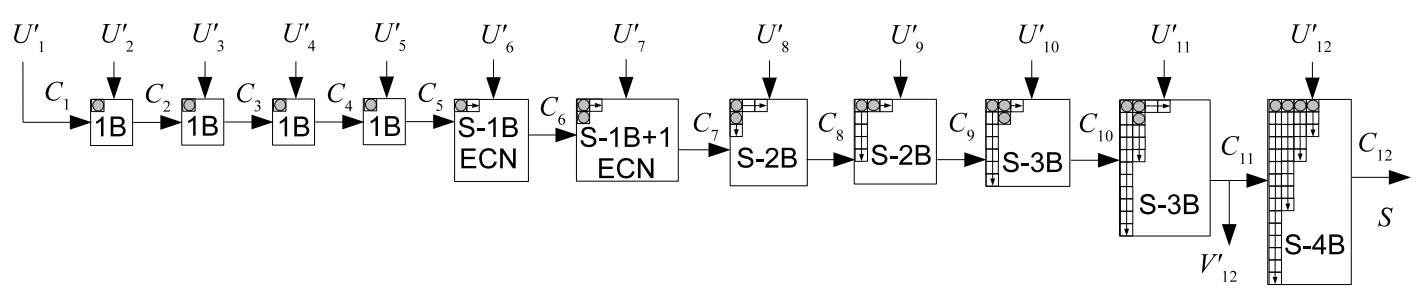

Fig. 7. Architecture of the proposed EF CN with $d_{c}=12, n_{b} \leq 4\left(n_{m, i n}=4\right), n_{c}^{12}=z=20$.

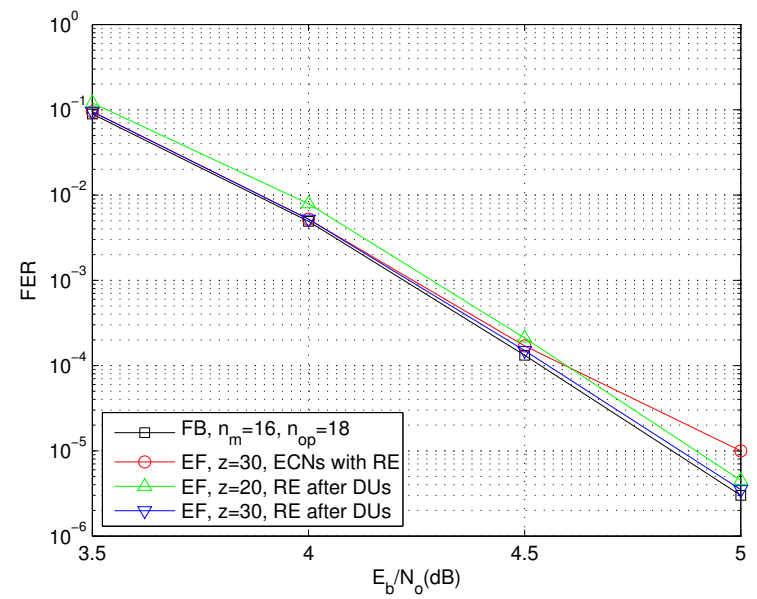

Fig. 8. Simulation results of NB-LDPC decoding algorithms for $(576,480)$ code over $\mathrm{GF}(64)$ and $d_{c}=12$ under AWGN channel.

discarded when complexity can be reduced without significant performance degradation.

After this pruning process, the structure of some ECNs is greatly simplified.

- 1B: only a single bubble is considered where $C_{i}$ is given by $C_{i}=\left\{\left(0, U_{i}^{\prime \oplus}[0] \oplus C_{i-1}^{\oplus}[0], C_{i-1}^{D}[0] \| 0\right)\right\}$ for $i>1$ and $C_{1}=\left\{\left(0, U_{1}^{\prime \oplus}[0] \oplus U_{2}^{\prime \oplus}[0], 00\right)\right\}$.

- S-1B: it generates directly the $n_{b}^{i}$ sorted output $C_{i}$ as $C_{i}=\left\{\left(0, U_{i}^{\prime \oplus}[0] \oplus C_{i-1}^{\oplus}[0], C_{i-1}^{D} \| 0\right),\left(U_{i}^{\prime+}[b], U_{i}^{\prime \oplus}[b] \oplus\right.\right.$ $\left.\left.C_{i-1}^{\oplus}[0], C_{i-1}^{D} \| 1\right)_{b=1 \ldots n_{b}^{i}-1}\right\}$. For this operation, a single $\mathrm{GF}$-adder is required.

- S-1B+1: only one comparator and one GF-adder are required.

- S- $x$ B: also known as S-bubble ECN. As described in [16], this architecture compares $x$ bubbles per clock cycle. It is composed of $x$ FIFO blocks, $x-1$ comparators, $x$ arithmetic adders and $x$ GF-adders, implemented with XOR gates.

Note that, as shown in Fig. 7, $V_{12}^{\prime}$ can be directly obtained from $C_{11}$.

\section{PERFORMANCE AND COMPLEXITY COMPARISON}

\section{A. Performance}

We consider bit-true Monte-Carlo simulations over the AWGN channel with a BPSK modulation scheme. Extrinsic and intrinsic LLR messages are quantified on 6 bits and $a$
TABLE II

POST-SYNTHESIS RESULTS FOR DIFFERENT ECN ARCHITECTURES AND ENTITIES ON A XILINX VIRTEX 6 FPGA.

\begin{tabular}{|c|c|c|c|c|c|c|}
\hline & \multicolumn{3}{|c|}{ Number of slices } & \multirow{2}{*}{$\begin{array}{l}F_{\max } \\
(\mathrm{MHz})\end{array}$} & \multirow{2}{*}{$\begin{array}{l}\text { Latency } \\
\text { (cycles) }\end{array}$} \\
\hline & & total & register & LUT & & \\
\hline \multirow{7}{*}{$\bigcup_{\text {Z }}^{\infty}$} & 1B & 7 & 25 & 7 & $>500$ & 1 \\
\hline & S-1B & 17 & 53 & 23 & $>500$ & 1 \\
\hline & $\mathrm{S}-1 \mathrm{~B}+1$ & 29 & 92 & 60 & 494 & 1 \\
\hline & S-2B & 49 & 89 & 112 & 338 & 1 \\
\hline & S-3B & 103 & 218 & 218 & 296 & 1 \\
\hline & S-4B & 117 & 157 & 275 & 319 & 2 \\
\hline & S-4B RE & 138 & 222 & 376 & 269 & 2 \\
\hline \multirow{5}{*}{ 氠 } & Sorter & 160 & 325 & 368 & 325 & 6 \\
\hline & Switch & 283 & 273 & 657 & $>500$ & 1 \\
\hline & DU & 21 & 55 & 14 & $>500$ & 1 \\
\hline & RE & 43 & 104 & 141 & 462 & 1 \\
\hline & Mult & 8 & 18 & 19 & $>500$ & 1 \\
\hline
\end{tabular}

posteriori LLRs on 8 bits. The maximum number of iterations is set to 10. Fig. 8 shows the Frame Error Rate (FER) performance of the $\mathrm{FB}$ and the $\mathrm{EF}$ algorithms for a $\mathrm{GF}(64)-$ LDPC code of size $(576,480)$. This code is regular with $d_{v}=2$ and $d_{c}=12$. As a reference, the FB decoder uses the S-bubble algorithm as in [16] with 4 bubbles, $n_{m}=16$ and $n_{o p}=18$, where $n_{o p}$ represents the number of outputs of an ECN before RE. The simulation EF CN with RE performed in ECNs shows a performance degradation and it shows that RE should not be done in ECN but after decorrelation as demonstrated in sub-section III-B and shown in Fig. 5 and Fig. 6. Simulation with $z=30$ shows negligible performance degradation. For the EF CN with $z=20$, the ECNs are as represented in Fig. 7 and simulation shows that performance versus complexity compromise can be found.

\section{B. Implementation results}

For hardware complexity evaluation, we implemented the different $\mathrm{CN}$ architectures and $\mathrm{CN}$ sub-unit on a Xilinx VIRTEX 6 FPGA device. The 6 types of ECNs used in PS EF CN represented in Fig. 7 and the 4S-bubble with RE used in FB $\mathrm{CN}$ were synthesized to obtain the results presented in Table II. The sorter and the switch (Fig. 4), the DU and the RE (Fig. 5) and the GF multiplier implementation results are also presented. For all implementations, the LLR and GF values are quantified on 6 bits. The latency in cycles (or number of pipeline stage) between input and output of each entity is also presented.

Table III summarizes the implementation results for the considered architectures for $\mathrm{GF}(64)$ and $d_{c}=12$. In some 
TABLE III

POST SYNTHESIS RESULTS FOR GF(64) CN ARCHITECTURES

\begin{tabular}{|c|c|c|c|c|c|}
\hline $\mathrm{CN}$ & \multicolumn{3}{|c|}{ Number of slices } & \multirow{2}{*}{$\begin{array}{l}F_{\max } \\
(\mathrm{MHz})\end{array}$} & \multirow{2}{*}{$\begin{array}{c}L_{C N} \\
\text { (cycles) }\end{array}$} \\
\hline $\mathrm{GF}(64)$ & Total & REG & LUT & & \\
\hline FB RE & 3904 & 6019 & 10314 & 227 & 22 \\
\hline PS FB RE & 2729 & 4119 & 6733 & 229 & 20 \\
\hline EF RE & 2633 & 4628 & 6768 & 217 & 24 \\
\hline PS EF RE & 1987 & 3151 & 4814 & 222 & 19 \\
\hline PS EF & 1495 & 2383 & 3616 & 227 & 19 \\
\hline
\end{tabular}

decoder implementations [9], [15], when code with $d_{v}=2$ are considered, the VNs are cascaded after the $\mathrm{CN}$ and a RE is performed in the VN. Since that in the PS EF architecture the RE block is performed at each $\mathrm{CN}$ output (as shown in Fig. 5 and Fig. 6), and is performed again in the VN, the RE can be removed from the $\mathrm{CN}$ output without performance loss. The EF shows significant complexity reduction compared with standard FB CN implementation. The PS EF CN with RE and without RE show 1.96 and 2.6 times less complexity than the standard FB CN, respectively.

$L_{C N}$ gives the latency in cycles of a $\mathrm{CN}$. For the $\mathrm{FB}$ architecture, $L_{C N}=L_{\text {mult }}+\left(d_{c}-2\right) \times L_{S-4 B}+L_{\text {div }}=22$. For PS EF architecture (Fig. 7), $L_{C N}=L_{\text {Sorter }}+L_{\text {Switch }}+$ $L_{E C N s}+L_{D U}+L_{\text {Switch }}=6+1+12+1+1=19$, considering that the multiplication is performed in the same cycle as the switch and the division is performed during the DU cycle.

\section{Thoughput}

High throughput can be obtained by processing $p$ CNs in parallel in a layered decoder [17], [9]. Because we focus on code with $d_{v}=2$, the VN update can be cascaded after the $\mathrm{CN}$ update as in [9], [15]. The throughput of the layered decoder is then given by $T(M b p s)=\left(K_{b} \times F_{\max } \times p\right) /\left(M \times P_{C N} \times\right.$ $\left.i t_{a v r}\right)$ where $p$ is the parallelism, $i t_{a v r}$ is the average number of iteration and $P_{C N}$ is the periodicity of the $\mathrm{CN}$ computations in a layered decoder. $P_{C N}$ in a layered decoder as in [9] is given by $P_{C N}=L_{C N}+L_{V N}+n_{m, i n}$ with $L_{V N}=3+$ $n_{m, \text { out }}+n_{m \text {,int }}$ where $n_{m, \text { int }}$ is the number of intrinsic values considered in the second phase of the VN [9]. The $(576,480)$ code allows a parallelism of up to 4 and simulation shown is Fig. 8 gives $i t_{a v r}=1.2$ at $\mathrm{SNR}=4.5$. With $n_{m \text {,int }}$ set to 4 , the throughput can reach $534 \mathrm{Mbps}$ with the FB architecture and 544 Mbps with the PS EF architecture.

\section{CONCLUSiON}

The presented solutions efficiently incorporate Elementary Check Nodes into a Syndrome-based architecture. Moreover the $\mathrm{CN}$ inputs are presorted leading to drastic simplification of most of the ECNs. The resulting architecture benefits from flexibility and linear complexity of the Extended Forward solution and complexity reduction due to presorting. The final architecture shows area reduction by up to a factor of 2.6 compared with standard FB decoder using S-bubble ECNs.

\section{REFERENCES}

[1] R. Gallager, "Low-density parity-check codes," Ph.D. dissertation, Cambridge, 1963.

[2] M. C. Davey and D. J. C. MacKay, "Low density parity check codes over GF(q)," in Information Theory Workshop, 1998, Jun 1998, pp. 70-71.

[3] D. Declercq and M. Fossorier, "Decoding algorithms for nonbinary LDPC codes over GF(q)," IEEE Transactions on Communications, vol. 55, no. 4, pp. 633-643, April 2007.

[4] A. Voicila, D. Declercq, F. Verdier, M. Fossorier, and P. Urard, "Lowcomplexity decoding for non-binary LDPC codes in high order fields," IEEE Transactions on Communications, vol. 58, no. 5, pp. 1365-1375, May 2010.

[5] Y. L. Ueng, K. H. Liao, H. C. Chou, and C. J. Yang, "A high-throughput trellis-based layered decoding architecture for non-binary LDPC codes using max-log-QSPA," IEEE Transactions on Signal Processing, vol. 61, no. 11, pp. 2940-2951, June 2013.

[6] E. Li, D. Declercq, and K. Gunnam, "Trellis-based extended Min-Sum algorithm for non-binary LDPC codes and its hardware structure," IEEE Transactions on Communications, vol. 61, no. 7, pp. 2600-2611, July 2013.

[7] P. Schläfer, N. Wehn, M. Alles, T. Lehnigk-Emden, and E. Boutillon, "Syndrome based check node processing of high order NB-LDPC decoders," in 22nd International Conference on Telecommunications (ICT), April 2015, pp. 156-162.

[8] P. Schläfer, V. Rybalkin, N. Wehn, M. Alles, T. Lehnigk-Emden, and E. Boutillon, "A new architecture for high throughput, low latency nbldpc check node processing," in Personal, Indoor, and Mobile Radio Communications (PIMRC), 2015 IEEE 26th Annual International Symposium on, Aug 2015, pp. 1392-1397.

[9] C. L. Lin, S. W. Tu, C. L. Chen, H. C. Chang, and C. Y. Lee, "An efficient decoder architecture for nonbinary ldpc codes with extended min-sum algorithm," IEEE Transactions on Circuits and Systems II: Express Briefs, vol. 63, no. 9, pp. 863-867, Sept 2016.

[10] H. Harb, C. Marchand, A. Ghouwayel, A. Conde-Canencia, L., and E. Boutillon, "Pre-sorted forward-backward NB-LDPC check node architecture," in 2016 IEEE International Workshop on Signal Processing Systems (SiPS), Oct 2016, pp. 142-147.

[11] V. Rybalkin, P. Schläfer, and N. Wehn, "A new architecture for high speed, low latency NB-LDPC check node processing for GF(256)," in 2016 IEEE 83rd Vehicular Technology Conference (VTC Spring), May 2016, pp. 1-5.

[12] C. Marchand and E. Boutillon, "NB-LDPC check node with pre-sorted input," in 2016 9th International Symposium on Turbo Codes and Iterative Information Processing (ISTC), Sept 2016, pp. 196-200.

[13] C. Poulliat, M. Fossorier, and D. Declercq, "Design of regular (2,dc)LDPC codes over GF(q) using their binary images," IEEE Transactions on Communications, vol. 56, no. 10, pp. 1626-1635, October 2008.

[14] A. Voicila, D. Declercq, F. Verdier, M. Fossorier, and P. Urard, "Lowcomplexity decoding for non-binary LDPC codes in high order fields," IEEE Transactions on Communications, vol. 58, no. 5, pp. 1365-1375, May 2010.

[15] E. Boutillon, L. Conde-Canencia, and A. A. Ghouwayel, "Design of a GF(64)-LDPC decoder based on the EMS algorithm," IEEE Transactions on Circuits and Systems I: Regular Papers, vol. 60, no. 10, pp. 2644-2656, Oct 2013.

[16] O. Abassi, L. Conde-Canencia, A. A. Ghouwayel, and E. Boutillon, "A novel architecture for elementary-check-node processing in nonbinary ldpc decoders," IEEE Transactions on Circuits and Systems II: Express Briefs, vol. 64, no. 2, pp. 136-140, Feb 2017.

[17] Y. L. Ueng, C. Y. Leong, C. J. Yang, C. C. Cheng, K. H. Liao, and S. W. Chen, "An efficient layered decoding architecture for nonbinary QCLDPC codes," IEEE Transactions on Circuits and Systems I: Regular Papers, vol. 59, no. 2, pp. 385-398, Feb 2012. 